\title{
REPLICACIÓN DE LA TÉCNICA DE SEPARACIÓN DE COMPONENTES EN MATERIAL CADAVÉRICO
}

\author{
María F. Ignatov Galan, Sofía Martínez Ventura, Daiana López Gil, Emilia Cerchiari \\ Campodónico, Eduardo Olivera Pertusso
}

\author{
Departamento de Anatomía, Facultad de Medicina. Universidad de la República, \\ Montevideo, Uruguay
}

\section{RESUMEN}

Introducción: la pared anterolateral del abdomen comprende 4 músculos: oblicuo externo, oblicuo interno, transverso abdominal y recto abdominal, que le permiten participar en funciones diversas. Las mismas pueden afectarse por una eventración. Cuando alcanzan grandes tamaños, su reparación puede representar un desafío para el cirujano general. La eventroplastia con malla irreabsorbible es el patrón oro para su tratamiento, pero este puede requerir de procedimientos adicionales. El objetivo del presente trabajo consistió en reproducir las técnicas de separación de componentes en material cadavérico, y realizar una revisión de la literatura. Material y Métodos: se utilizaron 2 cadáveres adultos, de ambos sexos, formolados. Se reprodujo la técnica de separación de componentes anterior mediante la liberación del músculo oblicuo externo. La separación de componentes posterior se realizó mediante labrado de un plano retro rectal y posterior progresión de la disección en sentido lateral. Resultados: Los colgajos generados permitieron un avance de la placa muscular medialmente. Conclusiones: el conocimiento anatómico de la pared anterolateral del abdomen es la base para la realización de las técnicas de separación de componentes. La reproducción en material cadavérico es un paso inicial en el entrenamiento quirúrgico de las mismas.

Palabras clave: Pared abdominal, anatomía, hernia incisional.

\section{ABSTRACT}

Introduction: the anterolateral abdominal wall includes 4 muscles: external oblique, internal oblique, transverse abdominis and rectus abdominis, which allow it to participate in diverse functions. These can be affected by an incisional hernia. When they achieve huge size, its repair may represent great challenge to the general surgeon. Eventhroplasty using nonabsorbable mesh is the gold standard treatment, but it can require additional procedures. Component separation technique is a group of anatomic-based procedures which allow the increase of abdominal volume. The aim of this report was to reproduce the component separation technique in cadaveric material, and a literature review. Materials and Methods: two adult cadavers of both sexes were used, fixed in formaldehyde. The external oblique muscle was liberated as described in the anterior component separation technique. Posterior component separation was made through a retro-rectal plane, and then progressing the dissection laterally. Results: Medial advancement of the muscles was achieved. Conclusions: knowledge of anterolateral abdominal wall anatomy is the basis for performing component separation technique. Training on cadaveric material is one of the first steps for surgical training.

Key Words: Abdominal wall, anatomy, incisional hernia.

\section{INTRODUCCIÓN}

La pared anterolateral del abdomen comprende 4 músculos: oblicuo externo, oblicuo interno, transverso abdominal y recto abdominal; extendidos desde el reborde costal al hueso coxal.

\section{* Correspondencia a: María F. Ignatov Galan. mariaignatov94@outlook.com}

Recibido: 2 de Abril, 2021. Revisado: 19 de Abril, 2021. Aceptado: 10 de Mayo, 2021. 
Según Kapandji (2006) cada músculo posee pequeños sectores musculares, anatómica y fisiológicamente independizados que le permiten participar en diversas funciones tales como: ventilación, maniobras de Valsalva, movimientos del tronco, y formación del conducto inguinal. Estas funciones pueden afectarse por una intervención quirúrgica y por la presencia de una eventración, que se define según Korenkov y Neugebauer (2001, p. 66) como: "cualquier defecto de la pared abdominal, asociado o no a una tumoración, que asienta sobre una cicatriz quirúrgica, que es perceptible o palpable al examen físico, o mediante un estudio de imagen". Constituyen sobre todo una complicación de los procedimientos quirúrgicos laparotómicos. Su frecuencia oscila entre el $3 \%$ $20 \%$ según Basta et al. (2019), Le HuuNho et al. (2012) y Burger et al. (2005). Su presencia se asocia a complicaciones agudas y crónicas, así como a una disminución de la calidad de vida del paciente, vinculada a la alteración que generan sobre la funcionalidad de la pared anterolateral del abdomen. La Sociedad Europea de Hernias las clasifica según su ancho en tres categorías: menores a $4 \mathrm{~cm}$, entre 4 y $10 \mathrm{~cm}$ y mayores a 10 cm (Muysoms et al., 2009).La reparación de éstas últimas, con pérdida de derecho a domicilio, representa un gran desafío para el cirujano general. Reintroducir el contenido y generar un adecuado cierre parietal sin tensión son los objetivos de este procedimiento (Hernandez, 2016). Respecto al uso de malla sintética, la eventroplastia ha demostrado disminuir significativamente la incidencia de recurrencia y complicaciones (López-Cano et al., 2018; Luijendijk et al. 2000; Tong et al., 2011). Por otra parte, la retracción y fibrosis parietal dificultan la reparación.

Para estos casos más complejos surge como recurso terapéutico la técnica de separación de componentes (SC). Descrita inicialmente por Ramirez et al. (1989), utiliza tejidos autólogos disecando la pared abdominal anterior a través de planos anatómicos, con el objetivo de lograr el cierre de la línea media. También se la ha denominado técnica de separación de componentes anterior (SCA). Los métodos de separación de componentes posterior (SCP) se fundamentan en la reparación retromuscular descrita inicialmente por Rives et al. (1985) y Stoppa (1989), con disección de la cara posterior del músculo recto anterior. Múltiples variantes de la técnica surgen en la evolución: técnicas laparotómicas como las descritas por Carbonell et al.(2008) y Novitsky et al. (2012) así como reparación mediante cirugía mini-invasiva.
El objetivo del presente trabajo consistió en reproducir en material cadavérico las técnicas de SCA y SCP. Además, se realizó una revisión no sistematizada de la literatura.

\section{MATERIAL Y MÉTODO}

Se utilizaron 2 cadáveres adultos pertenecientes al Departamento de Anatomía de la Facultad de Medicina de la Universidad de la República, Montevideo - Uruguay, de ambos sexos, fijados en solución de formaldehído al $10 \%$.

Se reprodujo la técnica de SCA descrita por Ramirez et al. (1989). Se realizó una incisión mediana xifopúbica, y se labraron extensos colgajos de piel y tejido celular subcutáneo hasta exponer la inserción medial del músculo oblicuo externo. Se localizó el borde externo del recto abdominal y $1 \mathrm{~cm}$ lateral a este se seccionó longitudinalmente la aponeurosis del oblicuo externo desde el reborde costal hasta el pubis y se liberó su cara profunda del músculo oblicuo interno. A través de la incisión mediana, se separó la cara profunda del recto abdominal de su vaina.

Para la SCP se reprodujo la técnica descrita por Carbonell et al.(2008). Se realizó una incisión mediana xifopúbica. Se incidió en la hoja posterior de la vaina del recto abdominal aproximadamente $1 \mathrm{~cm}$ lateral a la línea alba. Se labró así un espacio retromuscular; lateralmente se separó dicha vaina del músculo oblicuo interno. Esto permitió el acceso al plano entre oblicuo interno y transverso abdominal que se disecó. Posteriormente la hoja profunda de la vaina del recto abdominal se volvió a aproximar en la línea media con una sutura continua. Por último, se colocó una malla retro rectal prefascial y la vaina anterior del recto se volvió a aproximar en la línea media para cubrir la malla.

\section{RESULTADOS}

Ambas técnicas permitieron un avance medial de los colgajos miofasciales generados, permitiéndose el cierre sin tensión de los bordes aponeuróticos. El avance de los colgajos se estudió de forma cualitativa y no cuantitativa.

Se exhiben en material fotográfico los resultados obtenidos al practicar las disecciones descritas. En la Figura 1 se muestra la técnica SCA. En la Figura 2 se muestra la técnica SCP. 


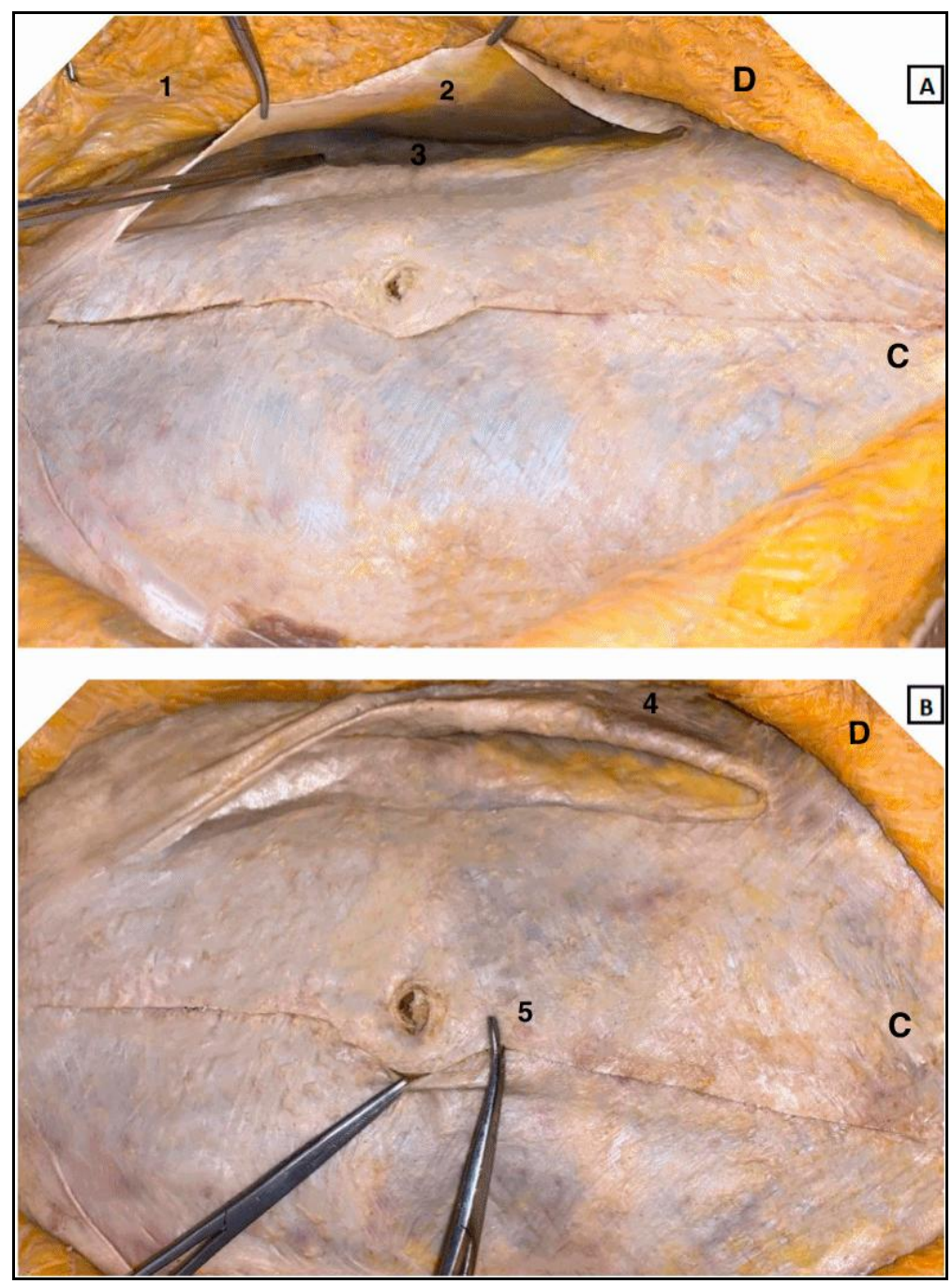

Figura 1.- SCA. Arriba (A) se muestran los extensos colgajos de piel obtenidos (1), la aponeurosis del músculo oblicuo externo (2) seccionada longitudinalmente, la cual se sostiene con instrumental y el músculo oblicuo interno en la profundidad (3). Abajo (B), se observa el músculo oblicuo externo retraído lateralmente (4) y al músculo recto abdominal (5) siendo avanzado en sentido medial formando un colgajo junto con oblicuo interno y transverso, luego de que se liberó la cara profunda del músculo oblicuo externo del músculo oblicuo interno. (D) derecha, (C) cefálico.

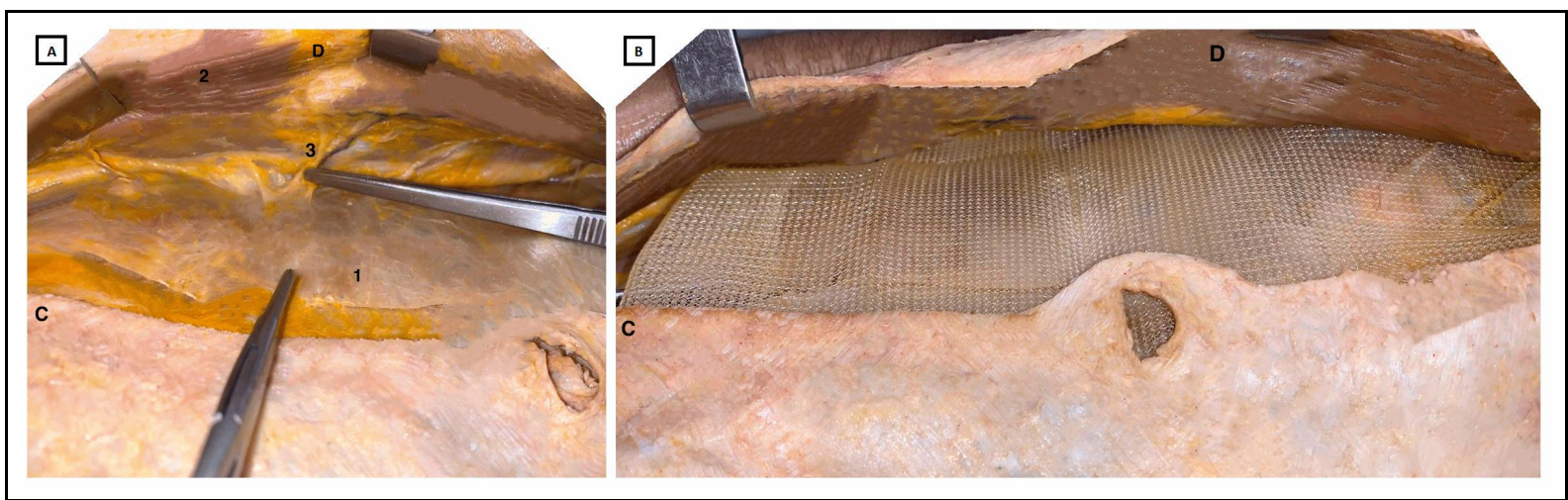

Figura 2.- SCP. A izquierda (A) se muestra el espacio retrorrectal. Se distingue: hoja posterior de la vaina del músculo recto abdominal (1), cara posterior del músculo recto abdominal (2), pedículo vasculonervioso de dicho músculo (3), abordándolo por su borde lateral. A derecha $(B)$ se visualiza la colocación de la malla de polipropileno en situación retromuscular pre-fascial. (D) derecha, (C) cefálico. 


\section{DISCUSIÖN}

Existen elementos de la anatomía de la pared anterolateral del abdomen que deben ser considerados para la realización de estas técnicas, ya sea porque facilitan su realización o la dificultan. Respecto a la SCA, Ramírez et al. (1989) destacan que la cara profunda del músculo oblicuo externo puede ser separada sencillamente de la cara superficial del músculo oblicuo interno puesto que se trata de un plano avascular. En segundo lugar, la cara profunda del músculo recto abdominal puede ser liberado fácilmente de la hoja posterior de su vaina siendo este plano también avascular.

En cuanto a la SCP la necesidad de colocación de grandes mallas para la reparación de defectos gigantes, ha llevado al diseño de técnicas que realizan una disección más allá del borde lateral de la vaina del recto. Ha sido ampliamente descrito que uno de los principales pedículos vasculonerviosos de la pared anterolateral del abdomen la abordan posteriormente para discurrir en el plano entre el músculo oblicuo interno y transverso, y luego alcanzar al músculo recto anterior por su borde lateral (Latarjet y Ruiz Liard, 2005; Testut y Latarjet, 1984). La SCP descrita por Carbonell et al. (2008), viola este plano, lo que constituye una limitante por el consecuente riesgo de denervación, atrofia y debilidad parietal. En este contexto Novitsky et al. (2012) describe la Técnica de liberación del músculo transverso abdominal (TAR por su sigla en inglés: transversus abdominis release), con el objetivo de preservar el aporte neurovascular lateral del recto abdominal. Esta variante consiste en, una vez liberada la cara posterior del recto abdominal, progresar la disección en sentido lateral, mediante la liberación del espacio entre el músculo transverso abdominal y la fascia transversalis y posterior aproximación del recto a la línea media. Se preserva así el paquete vasculonervioso lateral del músculo recto. A pesar de la notoria ventaja de dicha técnica, no fue posible reproducirla en material cadavérico formolado en nuestra experiencia previa, por la firme adherencia del peritoneo parietal y fascia transversalis que impiden la disección en este plano. Es por lo anterior, que no se incluyó en el actual trabajo. Podrá eventualmente realizarse en cadáveres no formolados.

Por otro lado, la SCP permite evitar los grandes colgajos de piel y tejido celular subcutáneo que se realizan en la SCA, con menor riesgo de seromas y hematomas según Heller et al. (2012) y Lowe et al. (2003) y menor riesgo de infección protésica y del sitio quirúrgico según Krpataet al. (2012). Estos hechos se explican por la preservación de la vasculatura dirigida al tejido celular subcutáneo. La variante mini-invasiva laparoscópica también presenta esta ventaja. Al momento de la elaboración del presente trabajo no se contó con la posibilidad de simulación laparoscópica en material cadavérico. Se optó por iniciar con las técnicas de SC abiertas, más difundidas en nuestro medio.

Según Trynor (2011) el uso de técnicas de simulación es beneficioso en la formación del cirujano debiendo estimularse su práctica. Ahmed et al. (2014) señalan mejoras significativas en el tiempo y seguridad al realizar los procedimientos, acortando así la curva de aprendizaje. La simulación cadavérica es una de estas técnicas, destacándose según Gilbody et al. (2011) y Reznick y MacRae (2006) por proveer una fiel representación de la anatomía quirúrgica. Según Carey et al. (2015) permite además el manejo de tejido auténtico y según Sugand et al. (2010), aporta una mejor comprensión de las dimensiones y relaciones de las estructuras. Como limitación, hay una menor complacencia tisular producto de los cambios post mortem y la fijación con formaldehído (Balta et al., 2019), limitación presente en nuestro estudio. Es por esto que no se realizaron medidas de tipo cuantitativo en el presente trabajo. Atribuimos a la misma razón la dificultad que encontramos en disecciones realizadas previamente para la liberación del músculo transverso abdominal.

La disección en material cadavérico fresco o en material cadavérico conservado mediante otras técnicas que preservan en mayor medida las características originales del tejido como la fijación en base a solución de Thiel, (Balta et al., 2019), se presentan como alternativas a ser consideradas para futuras investigaciones.

En conclusión, se lograron cumplir los objetivos de la investigación. Las mismas son una herramienta más en la reparación de eventraciones complejas y su reproducción en material cadavérico, es un paso inicial en el entrenamiento quirúrgico de las mismas. La realización de técnicas quirúrgicas en material de simulación contribuye a la curva de aprendizaje del cirujano en formación.

\section{Conflicto de intereses}

Los autores declaran no tener conflicto de intereses.

\section{Financiamiento}

Los autores declaran no haber recibido ningún tipo de financiación económica por parte de terceros.

\section{Aprobación Ética \\ Ninguna}




\section{Consentimiento informado}

Los cadáveres en los que se realizaron las disecciones, se obtuvieron de donantes voluntarios que expresaron su consentimiento de forma escrita, libre y voluntariamente, en vida. EI Departamento de Anatomía de Facultad de Medicina, Universidad de la República, cuenta con dichos consentimientos informados que habilitan a que dichos cadáveres sean usados para tareas de docencia e investigación.

\section{Contribuciones}

M.I Conceptualización, escritura, revisión y edición, investigación, redacción y borrador original. S.M Conceptualización, escritura, revisión y edición, investigación, redacción y borrador original. D.L Conceptualización, escritura, revisión y edición, investigación, redacción y borrador original. E.C Administración del proyecto, investigación, metodología, supervisión, validación, redacción y borrador original. E.O: Recursos.

\section{AGRADECIMIENTOS}

Los autores manifiestan su reconocimiento a todos quienes en vida deciden donar su cuerpo a nuestra Facultad para la docencia e investigación de la Anatomía.

\section{BIBLIOGRAFÍA}

Ahmed N, Devitt KS, Keshet I, Spicer J, Imrie K, Feldman L, Cools-Lartigue J, Kayssi A, Lipsman N, Elmi M, Kulkarni AV, Parshuram C, Mainprize T, Warren RJ, Fata P, Gorman MS, Feinberg S, Rutka J. 2014. A systematic review of the effects of resident duty hour restrictions in surgery: impact on resident wellness, training, and patient outcomes. Ann Surg. 259: 1041-53.

Balta JY, Twomey M, Moloney F, Duggan O, Murphy KP, O'Connor OJ, Cronin M, Cryan JF, Maher MM, O'Mahony SM. 2019. A comparison of embalming fluids on the structures and properties of tissue in human cadavers. Anat Histol Embryol. 48: 64-73.

Basta MN, Kozak GM, Broach RB. Messa CA, Rhemtulla I, DeMatteo RP, Serletti JM, Fischer $J P$. 2019. Can We Predict Incisional Hernia? Development of a Surgery-specific DecisionSupport Interface. Ann Surg 270: 544-53.

Burger JW, Lange JF, Halm JA, Kleinrensink GJ, Jeekel, H. 2005. Incisional Hernia: Early
Complication of Abdominal Surgery. World J. Surg 29: 1608-13.

Carbonell AM, Cobb WS, Chen SM. 2008. Posterior components separation during retromuscular hernia repair. Hernia 12: 359-62.

Carey JN, Minneti M, Leland HA, Demetriades D, Talving P. 2015 Perfused fresh cadavers: method for application to surgical simulation; Am J Surg 210: 179-87.

Gilbody J, Prasthofer AW, Ho K, Costa ML. 2011. The use and effectiveness of cadaveric workshops in higher surgical training: a systematic review. Ann R Coll Surg Engl 93: 347-52.

Heller L, McNichols CH, Ramirez OM. 2012. Component separations. Semin Plast Surg. 26: 25-8. 35.

Hernández A. 2016. Tratamiento actual de grandes eventraciones con las técnicas de separación de componentes anteriores y posteriores. Rev Hispanoam Hernia 4: 1-3.

Kapandji I. 2006. Fisiología Articular. Tomo 3. 6a Edición, Madrid: Editorial Médica Panamericana. 108-20.

Korenkov M, Neugebauer E. 2001. Classification and surgical treatment of incisional hernia: Results of an experts' meeting. Langenbeck's Arch Surg. 386: 65-73.

Krpata DM, Blatnik JA, Novitsky YW, Rosen MJ. 2012. Posterior and open anterior component separation: A comparative analysis. Am J Surg. 203: 318-22.

Latarjet M, Ruiz Liard A. 2004. Anatomía Humana. Tomo 2. 4a Edición, Buenos Aires: Editorial Médica Panamericana. 1305-30.

Le Huu Nho R, Mege D, Ouaïssi M, Sielezneff I, Sastre B. 2012. Incidence and prevention of ventral incisional hernia. J Visc Surg. 149: 314.

López-Cano M, Martin-Dominguez LA, Pereira JA, Armengol-Carrasco M, García-Alamino JM. 2018. Balancing mesh-related complications and benefits in primary ventral and incisional hernia surgery. A meta-analysis and trial sequential analysis. PLoS One.13: e0197813.

Lowe JB, Lowe JB, Baty JD, Garza JR. 2002. Risks Associated with "Components Separation" for Closure of Complex Abdominal Wall Defects. Plast Reconstr Surg. 111: 127683.

Luijendijk RW, Hop WC, van den Tol MP, de Lange DC, Braaksma MM, IJzermans JN, Boelhouwer $R U$, de Vries BC, Salu MK, Wereldsma JC, Bruijninckx CM y Jeekel J. 2000. A comparison of suture repair with mesh repair for incisional hernia. N Engl J Med. 343: 392-98.

Muysoms FE, Miserez $M$, Berrevoet $F$, Campanelli G, Champault GG, Chelala E, Dietz 
UA, Eker HH, El Nakadi I, Hauters $P$, Hidalgo Pascual M, Hoeferlin A, Klinge U, Montgomery A, Simmermacher RK, Simons MP, Smietański $M$, Sommeling $C$, Tollens $T$, Vierendeels $T$, Kingsnorth $A$. 2009. Classification of primary and incisional abdominal wall hernias. Hernia. 13: 407-14.

Novitsky YW, Elliott HL, Orenstein SB, Rosen MJ. 2012. Transversus Abdominis Muscle Release: A novel approach to posterior component separation during complex abdominal wall reconstruction. Am J Surg 204: 709-16.

Ramírez OM, Ruas E, Dellon AL. 1990. "Components Separation". Method for Closure of Abdominal-Wall Defects: An Anatomic and Clinical Study. Plast Reconstr Surg 86: 519-26.

Rives J, Pire JC, Flament JB, Palot JP, Body C. 1985. Treatment of large eventrations. NeW therapeutic indications apropos of 322 cases. Chirurgie, 111: 215-25.
Reznick RK, MacRae H. 2006. Teaching surgical skills - changes in the wind. N Engl J Med 355: 2664-69.

Stoppa RE. 1989. The Treatment Of Complicated Groin and incisional hernias. World J Surg 13: 545-54.

Sugand K, Abrahams P, Khurana A. 2010. The anatomy of anatomy: a review for its modernisation; Anat Sci Educ 3: 83-93.

Testut JL, Latarjet A. 1984. Tratado de Anatomía Humana. Tomo 2. 9 a Edición. Barcelona: Salvat editores. 352-54.

Tong WM, Hope W, Overby DW, Hultman CS. 2011. Comparison of Outcome After Mesh-Only Repair, Laparoscopic Component Separation, and Open Component Separation. Ann Plast. Surg 66: 551-56.

Traynor O. 2011. Surgical training in an era of reduced working hours: proceedings of the second international conference on surgical education and training. Surgeon. 9: S1-S2. 